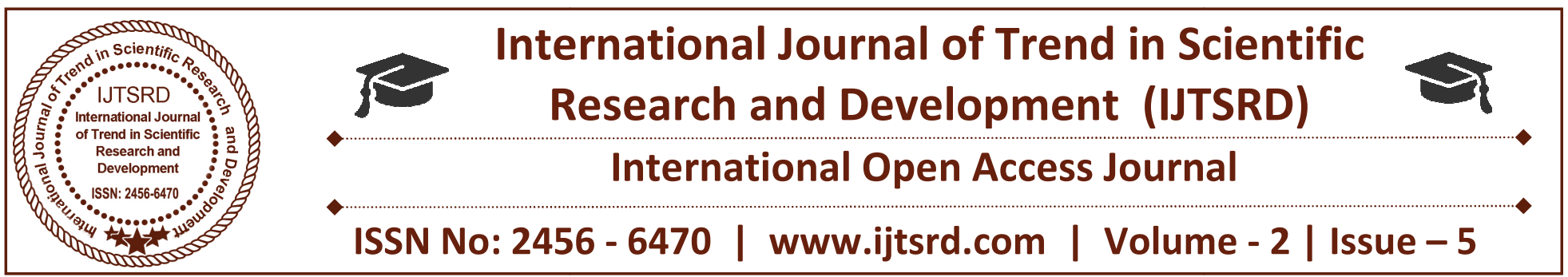

\title{
Study of Polyaniline Coated Fabrics for Electromagnetic Shielding Properties
}

\author{
N. Thukkaiappan \\ Lecturer Senior Grade, Department of Textile Technology, \\ Nachimuthu Polytechnic College, Pollachi, Tamil Nadu, India
}

\begin{abstract}
The interference of Electromagnetic among the appliances is one of the major problems to be solved at present. Electro-conductive media can generate and transport free charges, which can act as an electromagnetic shielding material. For light weight and flexible applications, here we prefer conductive polymer coating over textile instead of metals which are preferred traditionally. In this study electrically conductive cotton, polyester and polypropylene fabrics were made by coating polyaniline. The conductive fabrics were prepared by in-situ chemical oxidative polymerization of aniline using ammonium per sulphate as the oxidant by a process of diffusion polymerization in a mixed bath.. The surface resistivity study shows that the treated fabrics show better conductivity. There are no significant differences between the treated and untreated fabrics with respect to thickness, air permeability and stiffness test results. But washing and rubbing fastness results show significant degradation of coating with loss of conductive polymer.
\end{abstract}

Keywords: Polyaniline, Intrinsically conductive polymer, Electromagnetic Experiments for EMI SE

\section{INTRODUCTION}

Electrical and electronic systems in various fields emit electromagnetic radiations in various wavelength and frequencies. These radiations can interfere with other electronic devices and humans which can cause various problems. Electromagnetic shielding is a process of limiting the penetration of electromagnetic fields into a space, by blocking them with a barrier which must be a conductive material. Traditionally such shields are made of metallic materials as their

conductivity is higher. As metals are heavier and doesn't bend, it is better to go for textiles as they are flexible and light weight. But electromagnetic shielding property i.e. conductivity of the textiles is generally lower than metals. So to impart conductivity to a fabric there are many methods like metalizing the textile, carbon composites, coating conductive polymers, etc. Here we have chosen polyaniline which is an intrinsic conductive polymer. Out of the three commonly used conductive polymers, Polyaniline has the advantage of having the most inexpensive monomer, which is desired for commercial feasibility. Polyaniline has a complex structure which provides a possibility of creating conductive polymers with a diverse range of properties from chemical to electrical can be manipulated to produce materials with different conductivities. Polyaniline can be obtained either chemically or electrochemically.

\section{NEED OF STUDY}

In the past few years, the modernization in field of science and technology has created a new kind of pollution, i.e. electromagnetic interference (EMI). The advances in electronics reduce the component size and placing more number of electrical parts in limited space. Placing more number of components in a very confined space builds the problem of keeping electromagnetic interference of these systems from interfering with other systems through radiation. These radiations intentionally or unintentionally affect the activity of the other system, resulting in reduced performance or damage of the exposed systems. For example, interference of signal of television and 
sound speakers cause glimmering of picture as well as sound.

The human body is daily exposed to electromagnetic radiation of varying intensity at different places. The electromagnetic radiation causes damage to human cells which depends on frequency, intensity of electric and magnetic field, direction and polarisation of the waves. When these waves fall on human tissues, partially it gets transmitted. But most of the waves are absorbed by the body tissues which raise the core body temperature. When high frequency electromagnetic waves (range of radio frequency electromagnetic waves) enter an organism, it causes the molecules to vibrate, which produces heat and could obstruct a cell's capability for regeneration of DNA and RNA. Electromagnetic waves can cause abnormal chemical activities that produce cancer cell leading to leukaemia and other types of cancer. The network of veins in high risk organs such as eyes might be affected. This is due to heat build-up in the eyes by the electromagnetic waves which could not be easily dissipated. Prolong exposure to electromagnetic radiations increases the risk of heart problems, skin problems, head ache and many other diseases. Electromagnetic radiations can interfere with certain bio-electronic devices such as pacemakers, which are essential to the daily lives of affected people. Hence there is a great demand for electromagnetic shielding material.

\section{SHIELDING MECHANISM}

When electromagnetic rays pass through a medium or object then these rays interact with molecules of the medium or object. As the rays in form of wave impinge the surface of the object, it forces charges in the object to oscillate at same frequency of the incident wave. This forced oscillating charge behaves as an antenna and results in shielding phenomena. This can be divided into four different mechanisms namely reflection, multiple reflection, absorption and transmission. So in order to arrest the electromagnetic wave, it should be reflected or absorbed by the shielding materials.

\section{MATERIALS USED}

Pure cotton fabric, polyester fabric and polypropylene fabric were used for the polyaniline coating. The GSM of the fabrics were 122, 105 and 80 respectively. Aniline (monomer) Concentrated Hydrochloric acid (HCL-dopant) and Ammonium Peroxydisulfide (APS) (oxidizing agent).

\section{EXPERIMENTAL METHODS}

Conductive fabrics were made by in-situ chemical polymerization of aniline over the fabrics as mentioned earlier. In this process, freshly distilled $0.5 \mathrm{M}$ aniline was dissolved in the bath containing $0.35 \mathrm{~N}$ HCL solution for diffusion. After continuous stirring, the bath containing mixtures of aniline and aqueous acid were made to attain homogeneous mixture. The dry pre-weight fabric samples were placed inside this solution and allowed for 2 hours to react well with the monomer and dopant solution. Separately, 0.25M ammonium per sulphate (oxidizing agent) was dissolved in $0.35 \mathrm{~N}$ HCL solutions for polymerization. The oxidizing agent in the bath was then slowly added into the diffusion bath to initiate the polymerization reaction. The whole polymerization was carried out at room temperature for lhour. After completing the polymerization process, the polyaniline coated fabric was taken out and washed in water containing $0.35 \mathrm{~N} \mathrm{HCL}$ and later dried at $60^{\circ} \mathrm{C}$.

\section{TESTING STANDARDS}

\section{Air Permeability}

Air permeability is the volume of air in $\mathrm{cm}^{3}$ which passes in one second through one $\mathrm{cm}^{2}$ of fabric under a pressure head of $1 \mathrm{~cm}$ of water. Here,Air permeability is measured using air permeability tester. The resistance to air through fabric is noted and by using this value air permeability is measured.

\section{Stiffness}

Stiffness is a measure of resistance to bending. Bending length reflects the stiffness of a fabric when bent in one plane under the force of gravity. The Cantilever Test Option is the preferred procedure because it is simpler to perform. It is, however, not suitable for very limp fabrics. The specimens are cut to a size of 6inches $x$ 1inch. The specimens are cut in such a way that the warp specimens do not contain same warp yarns and weft specimens contain different weft yarns. Selvedges, creased or folded places must be avoided. The specimen is placed on the platform with template on top with their leading edges coincide. Both are pushed forward and sliding of specimen is stopped when it cuts index line.

\section{Rubbing Fastness}

Rubbing fastness test of fabrics coated with polyaniline were performed.. Each sample was abraded against cotton abrasive fabric for 10 cycles. Degradation of coating after rubbing fastness was 
evaluated by means of polyaniline fading and polyaniline staining according to the gray scale.

\section{Washing Fastness}

Washing fastness test was performed to test the resistance of the polyaniline coatings to physical actions. Washing test was performed according to the norm ISO 105-C06-2010. The standard conditions to be maintained to perform this test is $40^{\circ} \mathrm{C}, 30 \mathrm{~min}$. Degradation of polyaniline coating after this wash fastness test was evaluated by polyaniline fading according to the gray scale (ISO 105-A02).

\section{Electrical Resistivity}

The electrical resistance measurements were performed on all samples after conditioning the samples in a standard atmosphere. The resistance was measure ten times in each side of the sample and the average values were taken. The American association of textile chemists and colourists test method 76-1995 was used to measure the resistance of the samples and the surface resistivity of the fabric was calculated as follows

$$
\mathrm{R}=\mathrm{R}_{\mathrm{s}}(\mathrm{I} / \mathrm{w})
$$

Where, $\mathrm{R}$ is the resistance in ohms, $\mathrm{R}_{\mathrm{S}}$ is the sheet resistance or surface resistivity in ohms / square, $\mathrm{I}$ is the distance between the electrodes in $\mathrm{cm}, \mathrm{w}$ is the width of each electrode in $\mathrm{cm}$.

\section{TEST RESULTS}

\section{Air Permeability}

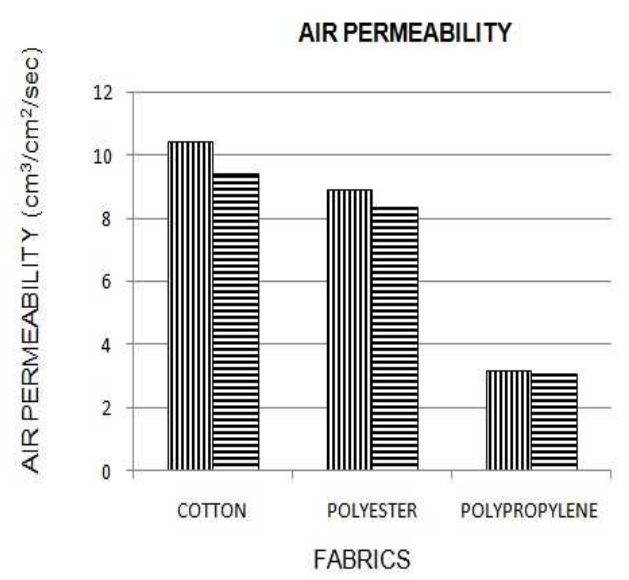

IIBEFORE COATING GAFTER COATING

The above graph shows the air permeability difference between the coated and uncoated fabrics. These values are obtained by converting the air resistance values to air permeability values. Since polyaniline being deposited over the fabric uniformly and polyaniline being penetrated into the fabrics the air permeability value decreases after coating.
Polypropylene shows lower air permeability value even before coating, which is due its fabric structure.

\begin{tabular}{|c|c|c|}
\hline \multirow{2}{*}{ FABRICS } & \multicolumn{2}{|c|}{$\begin{array}{r}\text { AIR PERMIEABILITY } \\
\left(\mathrm{cm}^{3} / \mathrm{cm}^{2} / \mathrm{sec}\right)\end{array}$} \\
\cline { 2 - 3 } & $\begin{array}{c}\text { BEFORE } \\
\text { COATING }\end{array}$ & $\begin{array}{c}\text { AFTER } \\
\text { COATING }\end{array}$ \\
\hline COTTON & 11.5 & 10 \\
\hline POLYESTER & 9.9 & 9.3 \\
\hline POLYPROPYLENE & 4.115 & 4.035 \\
\hline
\end{tabular}

Anova: Two-Factor Without Replication

\begin{tabular}{|c|c|c|c|c|c|c|}
\hline SUMMARY & Count & sum & Average & Variance & & \\
\hline Row 1 & 2 & 19.76 & 9.88 & 0.5 & & \\
\hline Row 2 & 2 & 17.2 & 8.6 & 0.18 & & \\
\hline Row 3 & 2 & 6.146 & 3.073 & 0.003528 & & \\
\hline Column 1 & 3 & 22.395 & 7.465 & 14.73948 & & \\
\hline Column 2 & 3 & 20.711 & 6.903667 & 11.53976 & & \\
\hline \multicolumn{7}{|l|}{ ANOVA } \\
\hline Source of Variation & SS & $d f$ & MS & $F$ & P-value & F crit \\
\hline Rows & 52.34759 & 2 & 26.17379 & 248.2277 & 0.004012 & 19 \\
\hline Columns & 0.472643 & 1 & 0.472643 & 4.482461 & 0.16845 & 18.51282 \\
\hline Error & 0.210885 & 2 & 0.105443 & & & \\
\hline Total & 53.03111 & 5 & & & & \\
\hline
\end{tabular}

From the analysis it is evident that there is no significant difference in air permeability between the coated and uncoated fabrics as $(4.48<18.51)$ null hypothesis is accepted, there is only minimal decrease in air permeability value but there is no significant difference. There is significant difference in air permeability between the fabrics as (248.2277 > 19). This variation is because that all the three fabrics are of different types and also different structures, the cover factors vary high leading to large variations in the results and thus these variations should not be taken into significant considerations as they are not of same specifications.

\section{Stiffness Results}

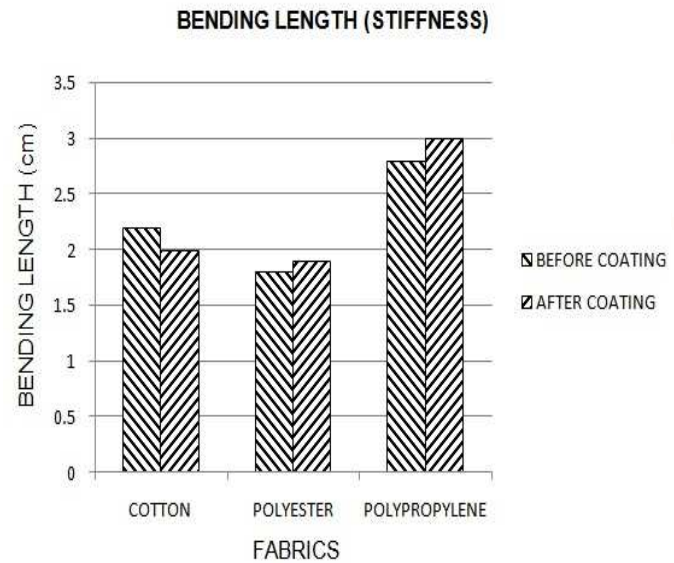


The above graph shows the stiffness difference between the coated and uncoated fabrics. It is surprising that cotton alone shows a different behaviour apart from the other two synthetic fabrics. For synthetic fabrics there is a general increase in bending length due to coating, but for cotton the uncoated fabric has higher bending length compared to polyaniline coated fabric. The reason behind this is that the polymer accumulates in fibre interstices or the polymer coating is thick enough it causes fibres to make contact with each other over a longer length. So it is evident that cotton fabric after coating was stiff as it deformed under its own weight. But for other two synthetic fabrics the uncoated fabrics were stiff which is due to their inherent in-extensibility property.

\begin{tabular}{|c|c|c|}
\hline \multirow{2}{*}{ FABRICS } & \multicolumn{2}{|c|}{ Bending Length $(\mathrm{cm})$} \\
\cline { 2 - 3 } & $\begin{array}{c}\text { BEFORE } \\
\text { COATING }\end{array}$ & $\begin{array}{c}\text { AFTER } \\
\text { COATING }\end{array}$ \\
\hline COTTON & 3.1 & 2.9 \\
\hline POLYESTER & 2.9 & 2 \\
\hline POLYPROPYLENE & 3 & 3.9 \\
\hline
\end{tabular}

Anova: Two-Factor Without Replication

\begin{tabular}{|l|r|r|r|r|}
\hline \multicolumn{1}{|c|}{ SUMMARY } & \multicolumn{1}{c|}{ Count } & \multicolumn{1}{c|}{ Sum } & Average & Variance \\
\hline Row 1 & 2 & 4.2 & 2.1 & 0.02 \\
\hline Row 2 & 2 & 3.7 & 1.85 & 0.005 \\
\hline Row 3 & 2 & 5.8 & 2.9 & 0.02 \\
\hline Column 1 & & & & \\
\hline Column 2 & 3 & 6.8 & 2.266667 & 0.253333 \\
\hline
\end{tabular}

\begin{tabular}{|c|c|c|c|c|c|c|}
\hline Source of Variation & SS & $d f$ & MS & $F$ & $P$-value & F crit \\
\hline Rows & 1.203333 & 2 & 0.601667 & 27.76923 & 0.034759 & 19 \\
\hline Columns & 0.001667 & $1>2>>$ & 0.001667 & 0.076923 & 0.80755 & 18.51282 \\
\hline Error & 0.043333 & 2 & 0.021667 & & & \\
\hline Total & 1248333 & & & & & \\
\hline
\end{tabular}

From the analysis it is explicit that there is no significant difference in stiffness values between the coated and uncoated fabrics as null hypothesis is accepted, there is only very little variations in bending length values but there is no significant difference. There is significant difference in stiffness between the fabrics as $(27.76>19)$. The reason behind this significant difference is that all these fabric structures are not similar and also due the individual property of the fabrics.

\section{Rubbing Fastness Results}

RUBBING FASTNESS

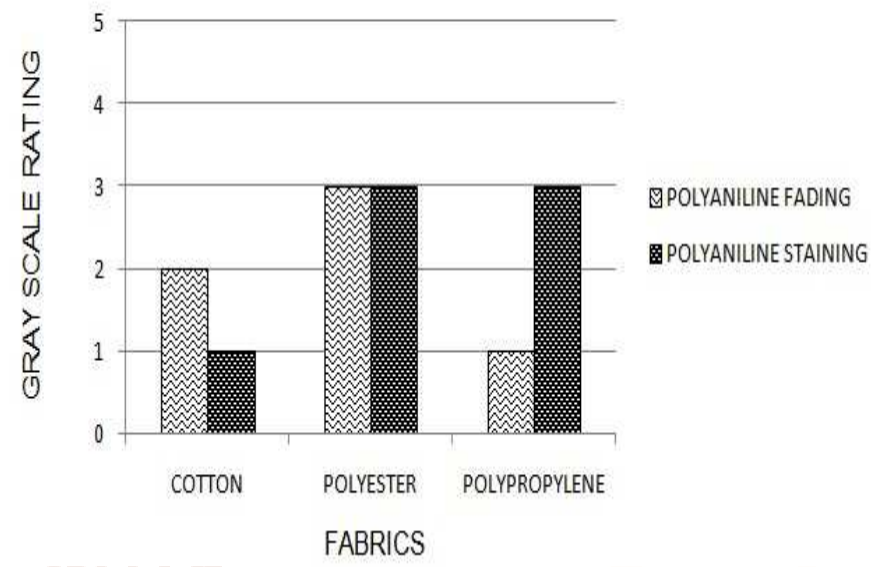

From the graph it is evident that there is degradation in coating with loss of part of the conductive polymer. Comparatively, polyester fabric had resistance against rubbing as polyaniline had better adherence with polyester. Polypropylene showed a very poor resistance against rubbing as after ten cycles, almost the whole coating was degraded.

\section{Washing Fastness Results}

\section{WASHING FASTNESS}

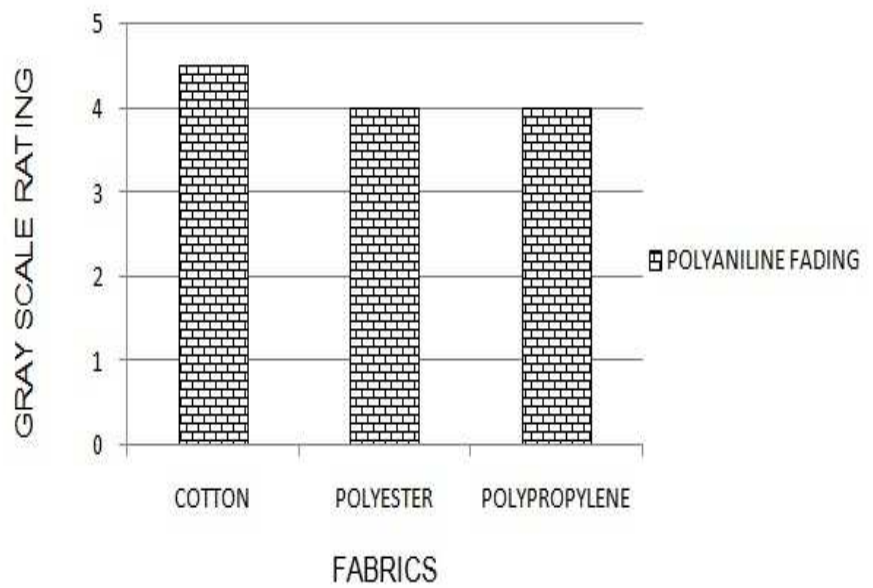

The wash fastness tests showed no degradation of coating, but still the fading values in gray scale is around 4.5-3. The reason behind this is because that the coated polyaniline fabrics with dark green colour were turned into blue shade. The reason for change in colour is due to soap. The soap employed produces a basic $\mathrm{pH}$ and causes de-protonation of polyaniline and consequently a colour change on the polyaniline coated fabric. 


\section{Electrical resistivity results}

ELECTRICAL RESISTIVITY

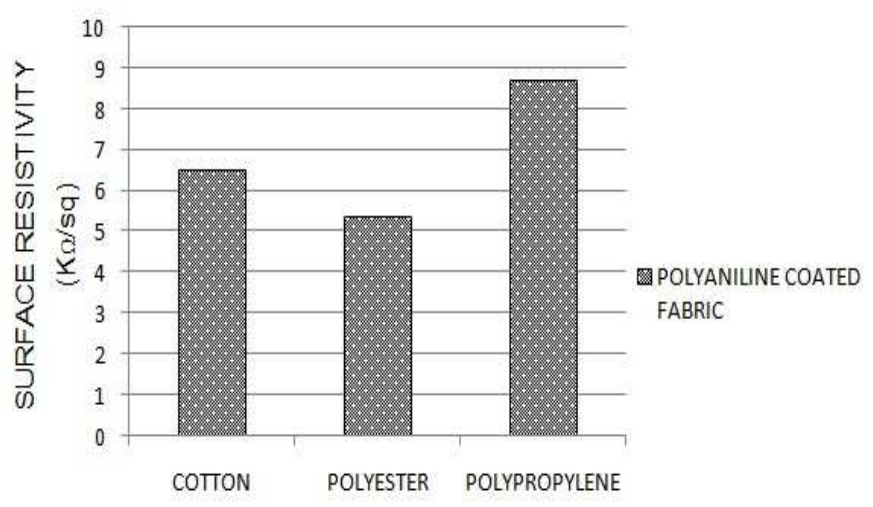

FABRICS conductive polymer coated fabrics can even be used in other areas.

The major conclusions of this work are, there is no significant difference in thickness, air permeability and stiffness properties between the coated and uncoated fabrics. The presence of polyaniline over the fabrics has increased the electrical conductivity of the fabrics.

\section{REFERENCES}

1. Anupama J. and Suwarna D., "Carbon Nanostructure Composite for Electromagnetic Interference Shielding", Pramana - Journal of Physics, Vol. 84, No. 6/2015, pp. 1099- 1116.

The electrical resistance values of the fabrics coated with polyanline is shown in the above graph. The results show that polyaniline can be coated over cotton, polyester and polypropylene fabrics and thus provide electrical conductivity. Comparatively polyester fabrics have lower resistance, so they can provide more conductances compared to other fabrics and can be best suitable for electromagnetic shielding applications.

\section{VIII.CONCLUSION}

Thus we have studied the basic properties and surface resistivity of electrically conductive polyaniline coated cotton, polyester and polypropylene fabrics. We knew that demand for conductive materials for electromagnetic shielding effectiveness is growing high as radiations have harmful effects. Electromagnetic shielding has wide range of applications that it can be used as curtain, canopies, etc., in versatile areas like, to prevent high frequency radiation from towers to enter living areas, to prevent interception of data from wireless network so that no outside signals can interfere inside providing high speeds, shielding of electromagnetic interference sensitive facilities and equipments, in military applications such as camouflage and radar protective fabrics for stealth technology, protection of sensitive technical equipments from radiation interference, packing materials for electronic devices, etc.,. These
2. Krishnasamy J., Alagirusamy R., Apurba D. and Ananjan B., "Electromagnetic Shielding Behaviour of Conductive Filler Composites and Conductive Fabrics - A Review", Indian Journal of Fibre and Textile Research, Vol. 39, No.

3. Gerard M., Chaubey A. and Malhotra B. D., "Application of Conducting Polymers to Biosensors", Biosensors and Bioelectronics, Vol. 17 , No. 5/2002, pp. 345-359.

4. Cochrane C., Vladan K., Maryline L. and Claude D., "Design and Development of a Flexible Strain Sensor for Textile Structures Based on a Conductive Polymer Composite", Sensors, Vol. 7, No. 4/2007, pp. 473-492.

5. Muthukumar N. and Thilagavathi G., "Development and Characterization of Electrically Conductive Polyaniline Coated Fabrics", Indian Journal of Chemical Technology, Vol. 19, No. 11/2012, pp. 434-441.

6. Devender T. S. and Narendera V. B., "Synthesis and Properties of Cotton Farics Modified with Polypyrole", Fibre, Volume 61, No. 2/2006, pp. 103-108.

7. Subhankar M., Kunal S., Pulak D. and Mrinal S., "Textiles in Electromagnetic Radiation Protection", Journal of Safety Engineering, Vol. 2, No. 2/2013, pp. 11-19. 9/2014, pp. 329-342. 